\title{
Research on the Assistant Driving System Based on Automobile Safety
}

\author{
Lijiao Liu ${ }^{1,3,}$, Hongyang Lu ${ }^{1}$, Yanyun Weng ${ }^{2}$, Ya Gao ${ }^{1,3}$, and Yuan Yu ${ }^{4}$ \\ ${ }^{1}$ China Transport Telecommunications \& Information Center, Beijing 100011,China \\ ${ }^{2}$ China Transport Telecommunication Information Group Co., Ltd. Beijing 100011, China \\ ${ }^{3}$ China Transportation Innovation Investment Development (Beijing) Co., Ltd. Beijing 100011, China \\ ${ }^{4}$ Research and Development Center of the Transport Industry for BeiDou Navigation Satellite System \\ Technologies and Applications, Beijing 100011, China
}

Keywords: ITS; Vehicle driving safety assistant system; Driving safety

\begin{abstract}
With the application of traffic safety to improve the level of attention and intelligent transportation system on the vehicle, a lot of vehicle driving safety assistant systems are put into use in the vehicle. Researching on road traffic safety protection technology based on intelligent transportation system is particularly urgent. Vehicle driving safety assistant system includes the driver monitoring warning system, collision avoidance system, cruise control system, the driver vision enhancement system. Installing vehicle driving safety assistant system is helpful to improve traffic safety. The use of information perception, dynamic identification, active safety control technology and method of improving the vehicle is one of the main research contents of ITS.
\end{abstract}

\section{System Composition}

With the rapid development of highway traffic, especially highway traffic, traffic accidents, especially malignant traffic accidents, are on the rise, and traffic safety is getting more and more attention. Therefore, the research of vehicle safety assistant driving system can provide safe assistant driving function for the vehicle, so as to provide intelligent technical guarantee for reducing traffic accidents caused by subjective factors of drivers[1].

The purpose of the research on the vehicle safety assistant driving system is to enable the vehicle to recognize the road condition information in the poor environment and assist the driver to drive safely. From the current development of vehicle safety assistant driving system, vision based environment perception, multi-sensor fusion, automatic driving and other technologies are its future development trend. Typical safety assistant driving systems include driver monitoring and warning system, collision avoidance system, cruise control system, driver vision enhancement system, etc.

\subsection{Driver monitoring and warning system}

Traffic accidents caused by driver fatigue account for a large proportion. According to the traffic accident death report of the highway traffic safety committee of the United States, it is estimated that nearly 8000 road traffic deaths each year are directly or indirectly caused by driver fatigue [2]. It can be seen that the problem of "fatigue driving" is an important factor affecting traffic safety.

Around the problem of fatigue driving, scholars at home and abroad have studied it from different perspectives. The existing fatigue monitoring methods are generally divided into three categories: the monitoring system based on the driver's external characteristics (such as eyelid movement, eye closure, nod movement, mouth opening and closing, etc.); the monitoring system based on the driver's physiological indicators measurement (such as EEG, ECG, skin potential and muscle activity, etc.) ; monitoring system based on driving behavior and vehicle parameters (such as steering wheel rotation frequency, throttle force, shift frequency, vehicle speed, acceleration, vehicle position).

\subsubsection{Monitoring system based on driver's external characteristics}

The "Toyota advanced safety car" developed by Toyota Motor Corporation of Japan adopts 17 
latest technologies, including the dozing driving alarm system. The principle is to monitor the movement of the driver's eyelids. Once the driver is found to be tired, the system first sends out a sound signal to the driver, and then sends out mint taste and cold air to wake up the driver.

\subsubsection{Monitoring system based on the driver's physiological indicators measurement}

The physiological index can reflect the weariness of people most accurately, among which EEG is called the "gold standard" to measure sleep. The fatigue warning device developed by Toyota Motor Company of Japan can measure the abnormalities if the driver is a little sluggish when operating the steering wheel, or if the pulse changes abnormally, and send a warning to make the cushion vibrate or brake automatically.

\subsubsection{Monitoring system based on driving behavior and vehicle parameters}

The driver's control ability to the vehicle will decline under the fatigue condition. The Spanish anti fatigue system monitors the driver's drowsiness by monitoring the steering pressure of the steering wheel. Once it is detected, the system warns the surrounding vehicles by flashing headlights and audible alarm, and automatically cuts off the fuel supply of the vehicle to stop the vehicle.

\subsubsection{Comparison of fatigue monitoring methods for drivers}

The advantages and disadvantages of several monitoring methods for driver fatigue are shown in Table 1.

Table 1 advantages and disadvantages of several monitoring methods for driving fatigue

\begin{tabular}{|c|c|c|}
\hline Monitoring method & Advantages & Disadvantages \\
\hline $\begin{array}{c}\text { Monitoring Method Based on } \\
\text { Driver's External } \\
\text { Characteristics }\end{array}$ & $\begin{array}{c}\text { The method is reliable, } \\
\text { especially the brain wave is the } \\
\text { "gold standard" for sleep } \\
\text { measurement, which can } \\
\text { provide the standard for driver } \\
\text { fatigue detection. }\end{array}$ & $\begin{array}{c}\text { This method needs some wires } \\
\text { or electrodes to contact the } \\
\text { driver's body and is not popular } \\
\text { with the driver. }\end{array}$ \\
$\begin{array}{c}\text { Monitoring Method Based on } \\
\text { the Driver's Physiological } \\
\text { Indicators Measurement }\end{array}$ & $\begin{array}{c}\text { Simple structure and mature } \\
\text { recognition algorithm. }\end{array}$ & $\begin{array}{c}\text { This method interferes with the } \\
\text { driver, so it is difficult to unify } \\
\text { the threshold value of } \\
\text { identifying fatigue state. For } \\
\text { example, the recognition rate is } \\
\text { not high due to the different } \\
\text { blink frequency. }\end{array}$ \\
\hline $\begin{array}{c}\text { Monitoring Method Based on } \\
\text { Driving Behavior and Vehicle } \\
\text { Parameters }\end{array}$ & $\begin{array}{c}\text { This method is easy to extract } \\
\text { signals and does not affect the } \\
\text { normal driving of drivers. }\end{array}$ & $\begin{array}{c}\text { Due to the influence of vehicle } \\
\text { type, road condition and } \\
\text { personal driving habits, the } \\
\text { recognition accuracy is not } \\
\text { high. }\end{array}$ \\
\hline
\end{tabular}

\subsection{Collision avoidance system}

The anti-collision system is an active driving aid system. Through the comprehensive perception of the environment inside and outside the cab, the obstacles around the vehicle, and the timely detection and alarm of dangerous situation, as shown in Figure 1, the driver or vehicle system can get enough safety time, so as to prevent or reduce the occurrence of collision.

Vehicle anti-collision system consists of three subsystems, namely, sensor sensing subsystem, which collects vehicle environment information; central processing subsystem, which evaluates traffic situation; output subsystem, which provides driver with driving information through humancomputer ecological interface, and timely controls vehicle through vehicle system in addition to driver's conscious response to adjust vehicle longitudinal and lateral control. The system diagram is 
shown in Figure 2.



Figure 1 Automobile collision avoidance system

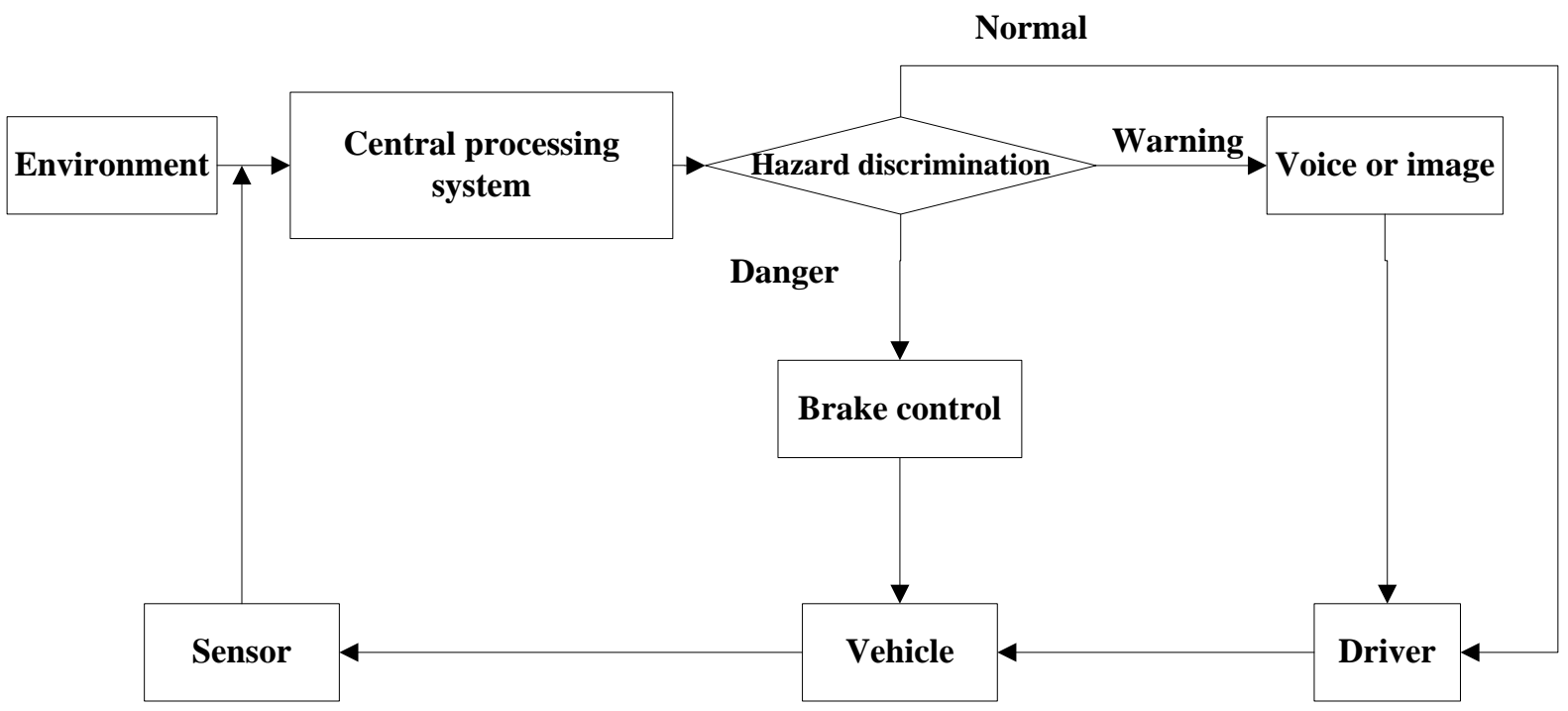

Figure 2 Structure of anti-collision system

The alarm system is mainly composed of radar, alarm controller and display control device [3]. The radar is mainly used to detect the distance, relative speed and other information between the car and the front car or obstacles; the alarm controller judges the driving safety state of the vehicle in real time based on the radar information and the moving state of the vehicle speed and so on; the display and control device displays the dangerous state information on the one hand, and on the other hand, the driver can set the key parameter values required by the alarm system. The alarm system realizes two alarm modes of hearing and vision. In the aspect of visual alarm, it can use both indicator light and graphic mode.

\subsection{Cruise control system}

The vehicle cruise control system is an auxiliary driving system that automatically adjusts the engine throttle under the precise control of the on-board control unit, so as to maintain the set speed under different road conditions. The main functional framework is shown in Figure 3.

Working principle of cruise control system: the driver sets the vehicle speed according to the needs, the sensor detects the real-time speed of the vehicle, the system automatically controls the throttle according to the difference between the set speed and the detection speed, according to a certain algorithm, so that the vehicle drives according to the set speed. The vehicle speed can be changed or temporarily cancelled during cruising, and then the cruising function can be restored [4]. 


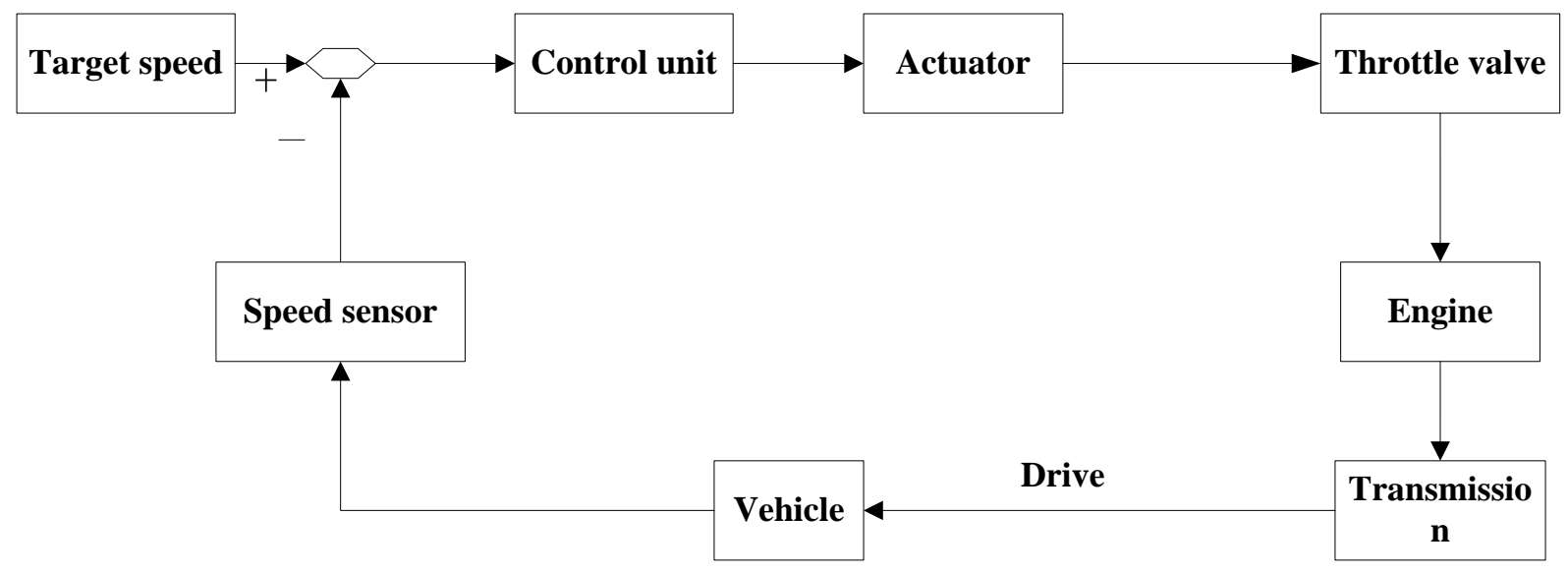

Figure 3 Structure of cruise control system

\subsection{Driver vision enhancement system}

Driver vision enhancement system can provide vision enhancement in different climate and different time of day, which is mainly composed of sensors, lighting system, recognition unit, information processing unit, information display screen, etc. The structure of visual enhancement system is shown in Figure 4.

\section{Driver display screen}

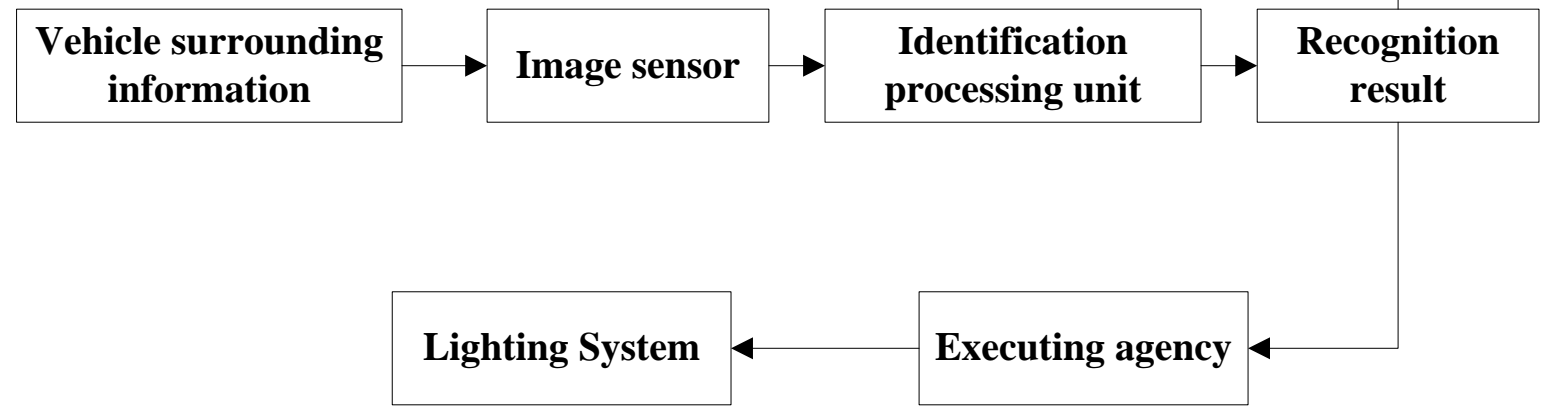

Figure 4 Composition diagram of visual enhancement system

The driver vision enhancement system monitors the road traffic environment through the sensor perception system, uses various image enhancement algorithms to enhance the visual effect, processes the visual information and obtains the real-time road traffic condition, the processed results can be displayed on the driver information display screen, and the driver can achieve the purpose of visual enhancement by watching the display screen.

\section{Application}

\subsection{Application in car}

If the safety assistant driving system is to be applied to the car, it must have high reliability. Therefore, relatively speaking, the application of new technology in this area is slow, and the implementation of various complex systems needs further study. For example, automatic driving technology, because it can provide higher safety, higher traffic efficiency, higher driving comfort, is concerned by many countries in the world. At present, many photoelectric products have been widely used in automobiles. For example, with the development of night vision technology, all kinds of low light and infrared night vision products have also entered the automobile market. Among them, the thermal imager has unique advantages different from other night vision devices. It can work in the fog, rain, snow and other bad weather, with a long acting distance and can identify 
camouflage and anti-interference. It has become a military night vision at home and abroad To some extent, it will replace LLL night vision instrument.

\subsection{Application in heavy truck}

With the rapid development of expressway, heavy vehicles have become the main type of road transportation. Due to the high tonnage of heavy vehicles, the transportation time is usually long, and most of them are day and night, so the traffic accidents of heavy vehicles on the highway are relatively frequent, resulting in huge losses. Therefore, a variety of products to improve the safety performance of vehicles have a broad market application prospect in heavy transport vehicles. According to statistics, there are about 50000 anti-collision systems currently used in heavy transport vehicles in the United States. Lane departure warning system products were first applied to heavy vehicles in 1999, and all kinds of night assistant driving systems have been applied to heavy trucks.

\subsection{Application in military vehicle}

In military and paramilitary fields, vehicle assistant driving system is widely used. In particular, night vision equipment and other night assistant driving systems are equipped on various military vehicles, which greatly improves the combat capability of the army at night. A large number of foreign tanks, armored vehicles and helicopters are equipped with night vision devices.

\section{Existing problems}

\subsection{Sensor is priced too high}

The integration of sensor technology is an important direction of the development of vehicle safety assisted driving technology. However, the high-precision radar, laser scanning instrument and other obstacle detection sensors are expensive, which affects their application.

\subsection{Misconceptions}

There are still some misunderstandings in people's understanding of vehicle safety assistant driving system. Most people think that with the vehicle safety assistant driving system, the driver can be completely liberated. In fact, this concept is impossible under the existing technical conditions. The current vehicle safety assistant driving system realizes the safe driving of the vehicle by interacting with the driver. The safe driving aid system only assists the driver to drive safely. Of course, with the further development of science and technology, it is not impossible to achieve complete machine driving.

\section{Conclusion}

Vehicle safety assistant driving system is a very active new field in the research of vehicle technology in the world, and vehicle driving safety guarantee technology is an essential and important part of it. Many countries in the world have invested a lot of human and material resources, and made many valuable research results. Some related technologies have been successfully applied to the driver assisted driving system, which provides strong technical support for reducing the traffic accidents caused by the subjective factors of the drivers of conventional vehicles, and produces obvious social and economic benefits.

China started the research in this field late, and limited by the level of infrastructure and economic strength and other factors, there is still a certain gap between the research in this field and the developed countries in the world. But this does not mean that our country will never do nothing in this field. Starting from China's national conditions, it is of far-reaching significance to conduct in-depth and detailed research in a certain field or some fields, track and catch up with the world's advanced level, and provide strong theoretical and technical support for the development of China's vehicle safety assurance technology and conventional vehicle safety assisted driving technology. 


\section{Acknowledgements}

Thanks to other scholars who provide theoretical and technical guidance for this paper!

\section{References}

[1] Nie Yao. Analysis of vehicle safety assistant driving technology. Traffic and transportation, 2008 (2): 146-147.

[2] Li Lingqi. Research on early warning technology of accident prone road section based on its. Chongqing: Chongqing Jiaotong University, 2011.

[3] Zhang Tiezhu, Jiang Hong. Intelligent target recognition based on airborne radar and infrared data fusion. Infrared and laser engineering, 2010,39 (4).

[4] Yan Xinping, Wu Chaozhong. Intelligent transportation system: principle, method and application. Wuhan: Wuhan University of Technology Press, 2014. 